\title{
Una aproximación general al transhumanismo y su problematización*
}

Antonio Luis Terrones Rodríguez

Recibido: 24 de enero de 2019 - Aprobado: 19 de febrero de 2019

El movimiento transhumanista está despertando un gran interés en las últimas décadas con sus polémicos postulados, y cuando sus propuestas son conocidas nadie queda indiferente ante estas. Diversos pensadores como Jürgen Habermas, Michael Sandel, Peter Sloterdjick o Nick Bostrom han escenificado una enriquecedora discusión en torno a los postulados transhumanistas. El transhumanismo defiende una mejora de la condición humana por medio de las tecnologías más avanzadas, entre las que se encuentran la biotecnología, la nanotecnología, la inteligencia artificial, etc. Son diversas las opciones para emprender el camino transhumanista: mejora de los hijos, mejora física, cognitiva y emocional, y lucha contra el envejecimiento. Además, las tecnologías de mejoramiento de la especie generan problemáticas de tipo social y político al insertarse en contextos con identidades sociales fuertemente marcadas, desigualdad económica y discriminación ética y racial. Así pues, es fundamental invitar a una reflexión ética sobre los postulados transhumanistas dentro de un marco democrático.

Palabras clave: transhumanismo, tecnologías, mejoramiento humano, ética.

* Artículo de reflexión. Elaborado en el marco del proyecto de investigación "Ontologías contemporáneas l", desarrollado por la Escuela de Filosofía de la Pontificia Universidad Católica del Ecuador y del que es director Stéphane Vinolo PhD. Citar como: Terrones, A. (2019). Una aproximación general al transhumanismo y su problematización. Análisis, 51(95), 319-345. DOI: https://doi.org/10.15332/21459169.4972

** Docente de la Escuela de Filosofía de la Pontificia Universidad Católica del Ecuador, Quito (Ecuador). Estudiante de doctorado en la Universitat de Valencia (España). Dirección postal: Facultad de Filosofía y Ciencias de la Educación, Av. Blasco Ibáñez 30, 46010 Valencia, España. Correo electrónico: antonioluis.terrones@gmail.com. Orcid: http://orcid. org//0000-0002-4015-8537 


\section{A general approach to transhumanism and its problematization}

Antonio Luis Terrones Rodríguez

\section{A bstract}

The transhumanist movement is awakening a great interest in recent decades with its controversial postulates and when its proposals are known no one is indifferent to them. Diverse thinkers as Jürgen Habermas, Michael Sandel, Peter Sloterdjick or Nick Bostrom have staged an enriching discussion around the transhumanist postulates. Transhumanism defends an improvement of the human condition through the most advanced technologies, among which are biotechnology, nanotechnology, artificial intelligence, etc. There are various options for undertaking the transhumanist path: improving children, physical, cognitive and emotional improvement, and fighting against aging. In addition, the enhancement technologies of the species generate social and political problems when inserting themselves in contexts with strongly marked social identities, economic inequality and ethical and racial discrimination. Therefore, it is fundamental to invite an ethical reflection on the transhumanist postulates within a democratic framework.

Keywords: transhumanism, technologies, human enhancement, ethics. 


\title{
Réflexions sur le trans-humanisme
}

\author{
Antonio Luis Terrones Rodríguez
}

\section{Résumé}

De par ses postulats et ses propositions, le mouvement trans-humaniste attire l'attention générale depuis une décennie. Des penseurs tels que Jürgen Habermas, Michael Sandel, Peter Sloterdijk ou Nick Bostrom ont participé à ce débat. Le trans-humanisme propose une amélioration de la condition humaine au travers des technologies les plus avancées, parmi lesquelles se trouvent la biotechnologie, la nanotechnologie, l'intelligence artificielle, etc. Divers sont les choix que l'on peut faire pour s'engager dans le chemin trans-humaniste : améliorer les enfants avant qu'il naissent, améliorer les aspects physique, moral, cognitive et émotionnelle, lutte contre le vieillissement. De plus, les technologies d'amélioration de l'espèce soulèvent des problèmes d'ordre social et politique puisqu'elles s'insèrent dans des contextes à identités très marquées, inégalité économique et discrimination éthique et raciale. Dès lors, il est fondamental d'entamer une réflexion éthique sur les postulats du trans-humanisme dans le cadre de la démocratie.

Mots clés: trans-humanisme, technologies, amélioration des êtres humains, éthique. 


\section{Introducción}

El ser humano puede ser considerado como homo sapiens, pero también como homo faber, y esas dos condiciones de ser determinan su modo de estar en el mundo. Existe una mutua imposición entre la naturaleza y el ser humano, el segundo le impone a la naturaleza, pero esta le condiciona de tal manera que se ve empujado a crear una sobrenaturaleza, como señalaría Ortega y Gasset (2004) en Meditación de la técnica.

Para el movimiento transhumanista, la ciencia actual, y en especial las disciplinas como la biología, la ingeniería genética, la nanotecnología, las neurociencias y también la computación, representan una buena oportunidad para mejorar (improvement) o potenciar (enhancement) la especie humana, y de ese modo generar seres humanos más fuertes físicamente, más inteligentes y emocionalmente más equilibrados. Para los transhumanistas esta mejora y potenciación de la especie humana no representa un sueño irrealizable o una utopía, sino más bien una realidad no tan lejana gracias al desarrollo de aquellas tecnologías que son necesarias para su realización.

Existen varias modalidades de transhumanismo, pero en este trabajo nos dedicaremos a estudiar el transhumanismo tecnocientífico, por entender que esa modalidad es la que se encuentra generalmente vinculada con la tecnología, y particularmente con la inteligencia artificial (IA). Además, el transhumanismo tecnocientífico se divide a su vez en dos vertientes, a saber, la que se encuentra más vinculada con el campo de la IA y la que tiene una base biológica y médica. En ese sentido, para hacer referencia a la vertiente más conectada con la IA, nos centraremos en pensadores como Raymond Kuzrweil y Nick Bostrom, entre otros; y para las que encuentran su sustento en el ámbito biológico y médico, haremos un mayor hincapié en las propuestas de Julian Savulescu, José Luis Cordeiro y David Wood. Al margen de esas consideraciones, también se dedicará un espacio de reflexión a otros pensadores que han promovido la reflexión y el debate sobre estas cuestiones, como Jürgen Habermas, Peter Sloterdijk, Michael Sandel y más recientemente Steven J. Jensen, José Luis Widow y Christine Overall, quien discute aspectos de índole social y política a raíz del fenómeno transhumanista. Por último, se hará una breve reflexión para argumentar la necesidad de someter a una valoración ética los postulados transhumanistas.

\section{Un nuevo paradigma}

El paradigma de la medicina tradicional, fundamentado en aspectos terapéuticos con el propósito de "reparar", ha sido cuestionado. En este nuevo escenario han surgido ideas como la de "mejoramiento" que están adquiriendo un notable interés. No obstante, el término "mejoramiento" presenta una doble problemática, a saber, la de exigir la determinación de qué es aquello que se está mejorando; y también respecto a qué estado es comparada la mejora (Feito, 2013, p. 269). Las 
controversias éticas en el ámbito de la mejora radican en el concepto de aquello que es considerado como mejor. Es una discusión sobre fines que son objeto de aquello que persiguen las biotecnologías promovidas por los transhumanistas, y además sobre los medios para alcanzar tales fines (Feito, 2013, p. 271). En ese sentido, el transhumanismo es un movimiento cultural e intelectual de carácter filosófico que se ha desarrollado en las últimas tres décadas, y como señalara Diéguez, es la utopía del momento (2017, p. 20).

El transhumanismo promueve un enfoque multidisciplinario y analiza las oportunidades de mejora de la condición humana y los organismos mediante el avance de la tecnología. Entre las disciplinas que tienen en cuenta para promover su proyecto están la ingeniería genética, la tecnología de la información, la nanotecnología o la IA. Los caminos para emprender la mejora se encuentran orientados a la salud, la cognición, la genética o el comportamiento emocional. El transhumanismo observa la naturaleza como una entidad susceptible de mejora y a los seres humanos como seres lo suficientemente inteligentes como para asumir la responsabilidad de mejorar. El transhumanismo sueña con alcanzar un horizonte poshumano, con capacidades mucho mayores que las que el ser humano presenta en la actualidad (Bostrom, 2003a; 2003b).

El enfoque terapéutico del paradigma médico tradicional encuentra su fundamento en una larga tradición judeocristiana (Diéguez, 2017), que hace que la reacción espontánea sea la de considerar la naturaleza humana como un elemento que tiene que ver con la "eternidad" y con lo "intangible", motivo por el que no es posible en ningún caso mejorar, sino curar o reparar. Esto se debe principalmente a que la tradición judeocristiana ha plasmado en el espíritu tradicional de Occidente una idea de oposición a la alteración o modificación de lo que consideran como la naturaleza humana. El transhumanismo se posiciona en contra de la postura tradicional judeocristiana, ya que se formula sobre la máxima de un perfeccionamiento ilimitado y un desafío a la muerte y el envejecimiento, entendiendo que la ciencia y la tecnología nos brindan las herramientas necesarias para esos fines.

El transhumanismo tiene influencias de la tradición del humanismo clásico y también de otras tradiciones en las que no cabe detenerse por falta de espacio en este momento, representada desde Pico della Mirandola, hasta Kant, pasando por Francis Bacon o Julien Offray de La Mettrie, quienes hacen hincapié en la idea de la perfectibilidad infinita del ser humano. Esa idea de perfectibilidad infinita es traducida en la máxima de perfeccionamiento humano ilimitado, defendida por el extropianismo, que es una filosofía transhumanista que fue presentada por Max More en 1990. Con el paso del tiempo la Declaración de principios transhumanistas fue modificada con las aportaciones de pensadores como Anders Sandberg, Max More, Natasha Vita-More, David Pearce, Leen Daniel Crocker, Mikhail Sverdlov y Nick Bostrom, entre otros. Finalmente, la organización internacional sin ánimo de lucro Humanity + adoptaría esta declaración en marzo de 2009 de la siguiente manera: 
1. La humanidad se verá profundamente afectada por la ciencia y la tecnología en el futuro. Prevemos la posibilidad de ampliar el potencial humano superando el envejecimiento, las deficiencias cognitivas, el sufrimiento involuntario y nuestro confinamiento al planeta Tierra.

2. Creemos que el potencial de la humanidad aún no se ha realizado. Hay posibles escenarios que conducen a condiciones humanas mejoradas maravillosas y que merecen la pena.

3. Reconocemos que la humanidad enfrenta graves riesgos, especialmente por el mal uso de las nuevas tecnologías. Existen posibles escenarios realistas que llevan a la pérdida de la mayoría, o incluso de todo, lo que consideramos valioso. Algunos de estos escenarios son drásticos, otros son sutiles. Aunque todo progreso es cambio, no todo cambio es progreso.

4. Es necesario invertir el esfuerzo de investigación para comprender estas perspectivas. Necesitamos analizar cuidadosamente la mejor manera de reducir los riesgos y acelerar las aplicaciones beneficiosas. También necesitamos foros donde las personas puedan discutir de manera constructiva lo que se debe hacer y un orden social donde se puedan implementar decisiones responsables.

5. La reducción de los riesgos existenciales y el desarrollo de medios para la preservación de la vida y la salud, el alivio del sufrimiento grave y el mejoramiento de la previsión y la sabiduría humanas deben perseguirse como prioridades urgentes, y contar con una gran financiación.

6. La formulación de políticas debe guiarse por una visión moral responsable e inclusiva, tomando en serio las oportunidades y los riesgos, respetando la autonomía y los derechos individuales, y mostrando solidaridad y preocupación por los intereses y la dignidad de todas las personas en todo el mundo. También debemos considerar nuestras responsabilidades morales hacia las generaciones que existirán en el futuro.

7. Abogamos por el bienestar de toda la sensibilidad, incluidos los humanos, los animales y cualquier futuro intelecto artificial, formas de vida modificada u otras inteligencias a las que pueda dar lugar el avance tecnológico y científico.

8. Estamos a favor de permitir a los individuos una amplia elección personal sobre cómo habilitan sus vidas. Esto incluye el uso de técnicas que pueden desarrollarse para ayudar a la memoria, la concentración y la energía mental; terapias de extensión de la vida; tecnologías de elección reproductiva; procedimientos crionicos; y muchas otras posibles tecnologías de modificación y mejora humana (Humanity +, 2018). 


\section{Posibles caminos transhumanistas}

El despliegue de la agenda práctica que promueve la declaración de principios del transhumanismo se apoya en los importantes avances que el campo científico y tecnológico han experimentado en las últimas décadas: biotecnología, nanotecnología, tecnologías de la información y ciencias del conocimiento. Entre las aplicaciones de estas cuatro áreas convergentes entre sí, nos podemos encontrar: la fabricación de nuevos materiales industriales que tienen más resistencia para la elaboración de prótesis, la elaboración de productos alimenticios y de supervivencia que aumentan el rendimiento energético, la investigación destinada a la creación de nanorobots para la reparación de tejidos y organismos, diseño de proteínas que ayuden a combatir enfermedades, etc. Y también otra serie de mecanismos que mejoren nuestras capacidades cognitivas y sensitivas, como la visión nocturna o el aumento de la potencia memorística. La última promesa en la que se está investigando trata sobre la creación de mecanismos que permitan la interacción cerebro-máquina, con el objetivo de trascender la inteligencia biológica y caminar hacia un terreno tecnológico. En este último aspecto tiene una notable influencia la inteligencia artificial. El interés en la aplicación de las cuatro áreas convergentes anteriormente mencionadas tiene como finalidad la superación de la especie humana y la construcción de un futuro poshumano que se materializa en los ámbitos que se presentan a continuación.

\section{La mejora de los hijos}

El transhumanismo consagra como una obligación moral (Cordeiro y Wood, 2018; Savulescu, 2012) y un derecho de los padres y madres la posibilidad de procrear los mejores hijos. Esta obligación moral y derecho es considerado por Julian Savulescu (2012), una "beneficiencia procreativa", que describe de la siguiente manera:

Las parejas (o los reproductores individuales) deberían seleccionar, de los distintos niños que pueden tener, aquel que se espera que tenga una vida mejor, o al menos una vida igual de buena que los demás, según la información relevante de la que se disponga.

Defenderé que la Beneficiencia Procreativa implica que las parejas deben emplear pruebas genéticas para rasgos no patológicos a la hora de seleccionar qué niño traer al mundo y que debemos permitir que se seleccionen genes no patológicos incluso si hacerlo así mantiene o incrementa la desigualdad social.

El "deberían" que aparece en "deberían seleccionar", ha de entenderse como "tiene buenas razones para". Entenderé que la moralidad nos exige que hagamos aquello que tenemos mejores razones para hacer. En ausencia de alguna otra razón, una persona 
que tiene una buena razón para tener el mejor niño posible está requerida moralmente a tenerlo. (Savulescu, 2012, pp. 45-46)

Fermín Jesús González Melado diferencia entre dos tipos de eugenesia, la negativa y la positiva (2010, pp. 211-212). La eugenesia negativa consiste en la eliminación de los fetos que presentan patologías a través de un diagnóstico prenatal o la interrupción del embarazo. Este mecanismo trata de evitar las patologías con el interés de seleccionar gametos en función de las características genéticas que presentan. En la eugenesia positiva se promueve el mejoramiento del niño por medio de una ingeniería genética que posibilitará "no solo la identificación de genes defectuosos, sino también la identificación de genes que expresen características deseables, por ejemplo, color de ojos, estatura, peso, inteligencia... Se trata de construir el mejor hijo posible" (González, 2010, p. 212).

A diferencia de González Melado, Savulescu proporciona una visión optimista de la selección genética haciendo un mayor hincapié en el aspecto de la inteligencia, pues la considera un motivo más que suficiente para decantarse por la posibilidad de seleccionar.

\section{La mejora física}

Otro de los caminos hacia el terreno poshumano es el de la mejora del rendimiento, con unas profundas aspiraciones hacia la superioridad de la especie. Este camino promueve el abandono de los mecanismos terapéuticos y defiende la incorporación de los fármacos o drogas para aumentar el rendimiento físico o memorístico de nuestra especie. El ámbito deportivo es visto por las teorías transhumanistas como un terreno fértil, en el que es posible hacer realidad muchas de las ideas que defiende este movimiento. El motivo por el que el ámbito deportivo se convierte en un objeto de estudio de las teorías del mejoramiento es por la combinación que existe entre el empeño, la admiración y la excelencia, que representan, en términos generales, un ideal para la vida buena. La biotecnología, la farmacología y la genética convierten la búsqueda del rendimiento superior en una de sus prioridades para la mejora física. El campo deportivo se convierte así en un espacio ideal para la investigación y experimentación de las tesis transhumanistas.

Savulescu (2012) entiende que es razonable defender el uso de las drogas en el deporte, ya que afirma que el ideal deportivo vinculado con la fortaleza biológica no es incompatible con nuestra capacidad de juicio y razonamiento. Para ello argumenta que no es pertinente sostener el ideal deportivo mediante la comparación con la actividad deportiva que desarrollan ciertos animales como los caballos. Los humanos no son caballos, nosotros tomamos decisiones y las hacemos con base a nuestra capacidad de razonamiento y juicio, algo que nos diferencia a grandes rasgos del resto de los animales. Esta diferencia, frente a quienes sostienen las tesis que se oponen al uso de drogas en el ámbito deportivo, refuerza una vez más el espíritu humano integrado en el deporte, ya que 
“lejos de ir contra el espíritu del deporte, la manipulación biológica encarna el espíritu humano: la capacidad de mejorarnos a nosotros mismo basándonos en nuestra razón y nuestro juicio" (Savulescu, 2012, p. 111).

Torbjörn Tännsjö (2017) reconoce que el ámbito deportivo cuenta con unos valores intrínsecos que lo hacen particular, y eso es lo que permite establecer una clara diferencia entre la medicina deportiva y la medicina en general. Mientras que en la medicina general las medidas positivas para el mejoramiento cuentan con cierto reconocimiento en el campo en cuestión, la medicina deportiva observa con cierto recelo las motivaciones que se esconden detrás de los mecanismos de mejoramiento. El deporte de élite cuenta con una serie de valores y una noción muy especial de justicia que lo hacen merecedor de cierto reconocimiento. En ese sentido, Tännsjö entiende que esos aspectos deben protegerse y quedar al margen de las modas de la técnica de mejoramiento, aunque, al mismo tiempo, reconoce que será difícil que el deporte se mantenga al margen de los avances tecnológicos en esta materia (Tännsjö, 2017, p. 338).

\section{La lucha contra el envejecimiento}

Una obra en la que se refleja una interesante reflexión sobre el asunto del envejecimiento es La muerte de la muerte de José Luis Cordeiro y David Wood. Es importante partir de la consideración de que el envejecimiento no es un proceso unitario dentro del cuerpo humano, pues hay organismos que no se someten a dicho proceso. Además, son múltiples los factores que influyen en el envejecimiento, como el medio ambiente. No obstante, a pesar de los estudios que existen en este campo, todavía no existe una teoría aceptada por parte de toda la comunidad científica acerca del envejecimiento. En medio de esta falta de consenso, el tecnólogo Aubrey de Grey, junto con Michael Rae, publicaron una obra titulada El fin del envejecimiento. Los importantes estudios formativos por los que había pasado De Grey, sobre todo en el campo de la informática y la computación, le aportaron una visión sobre el tema del envejecimiento muy peculiar.

Su postulado sobre la extensión de la vida se llama SENS (Strategies for Engineered Negligible Senescence; y se traduce al español como: estrategias para la senescencia negligible ingenierizada). El significado de este postulado trata sobre la posibilidad de desarrollar terapias médicas que tengan como objetivo combatir el envejecimiento en humanos. Dichas terapias médicas se deben plantear desde la bioingeniería, un campo que permitirá combatir las causas y daños del envejecimiento. La propuesta de De Grey no estuvo exenta de críticas y cuestionamientos, pues el mismo Massachusetts Institute of Technology (MIT) promovió un número especial de su revista Technology Review y un premio de 20.000 dólares para quien pudiera desmontar la teoría SENS. A pesar de las dudas que despertó en la comunidad científica este postulado teórico, los avances científicos han demostrado que De Grey estaba en lo cierto. 
A pesar de las diferencias entre las investigaciones de la comunidad científica, se presentan dos puntos básicos en los que existe ciertamente un consenso: que el envejecimiento se da de manera gradual, siendo un proceso dinámico y secuencial; y que no es considerado como algo inevitable o irreversible, ya que es un proceso plástico y flexible (Cordeiro y Wood, 2018, p. 89). Este consenso ha permitido comenzar a identificar el envejecimiento como una enfermedad, lo que abre un abanico de posibilidades para su tratamiento. Para Cordeiro y Wood (2018, p. 95), esto permite situar el envejecimiento en el centro de nuevas investigaciones, lo que favorece la búsqueda de fuentes de financiación y a la vez sugiere a que en los próximos años todo apunta para la creación de un nuevo nicho industrial.

\section{La mejora cognitiva y emocional}

En el texto Why I Want to be a Posthuman When I Grow Up, Nick Bostrom sostiene que un poshumano es aquel ser que tiene al menos una capacidad poshumana, entendiendo esto como la capacidad central general que excede en gran medida el máximo que puede alcanzar cualquier ser humano sin someterse a las mejoras tecnológicas (Bostrom, 2008, p. 107). Entre esas capacidades centrales generales distingue tres: las pertenecientes a la salud, la cognición y la emotividad. En este apartado nos centraremos en la cognitiva y la emotiva.

Bostrom afirma que existe un deseo generalizado en todos los seres humanos para mejorarse, pues, según él, todo el mundo quiere ser mejor, a nadie le disgusta mejorar. En lo que refiere a la cognición, entiende que las personas también están interesadas en mejorar su conocimiento y su inteligencia. Una clara muestra de ello son las grandes sumas de dinero que destinamos al cultivo del conocimiento, tanto si lo observamos desde plano individual o colectivo. Las personas se inscriben en académicas de artes musicales y también en centros que sirven para reforzar aquellos conocimientos que se aprenden en la escuela o la universidad. En ese sentido, para Bostrom (2008, p. 115) es más que evidente que nos preocupamos por la mejora de nuestro funcionamiento cognitivo. La mejora cognitiva tiene por sí misma un valor intrínseco, aunque también puede convertirse en un medio para la obtención de bienes externos como el honor, la fama o el dinero. Por lo tanto, una buena razón para defender una mejora cognitiva es el valor intrínseco que representa (Bostrom, 2008, p. 116).

A diferencia de la mejora en el ámbito de la salud, es más difícil tratar de explicar en qué consiste la mejora emotiva. Bostrom afirma que normalmente es más fácil entender la posibilidad de ayudar a alguien que sufre una depresión suicida como resultado de un desequilibrio neuroquímico para que disfrute de la vida, que aquellos casos que van más allá de las intervenciones terapéuticas que tratan de curar psicopatologías. En la mayoría de los casos que van más allá de lo estrictamente terapéutico para la cura de psicopatologías es muy difícil determinar en qué consiste un nivel de capacidad emocional poshumana (Bostrom, 2008, p. 117). 
Dedicamos gran parte de nuestra vida a tratar de forjar un carácter y moldearlo según nuestras necesidades. En ocasiones tratamos de vencer el miedo y la desconfianza por medio de algunos ejercicios. Así pues, tratamos de alcanzar aquellas metas que para nosotros implican una modificación y mejora de nuestras capacidades emocionales. La tecnología representa una potente herramienta que posibilitaría una mejora emocional poshumana. Existen capacidades emocionales que podrían estar orientadas hacia la excelencia gracias a la tecnología. Además, los medios tecnológicos inducirían la aparición de un nuevo escenario en el que florecieran nuevas emociones y estados psicológicos completamente nuevos, aunque esto no queda exento de dificultades a la hora de la comprensión (Bostrom, 2008, p. 118). Por lo tanto, las mejoras cognitivas y emocionales se presentan como un atractivo viable dentro de las opciones transhumanistas.

\section{Un deber moral controvertido}

La declaración de principios que promueve el transhumanismo ha generado un choque de perspectivas entre aquellos que piensan que la mejora de la especie humana es una obligación moral y aquellos que la cuestionan desde diversos enfoques. Así pues, aquí se tratará de dar cuenta con brevedad de esas dos perspectivas claramente enfrentadas, desde las aportaciones de dos pensadores que consiguen aglutinar un eje discursivo sin necesidad de recurrir a la diversidad de tesis que vienen de uno y otro lado.

John Harris (2017) es un firme defensor de las técnicas de mejoramiento por medio de las biotecnologías. Entiende que las técnicas de mejoramiento son buenas para los seres humanos porque permiten dejar atrás un estado que no suponía una mejora real. Existen razones indiscutibles para defender el mejoramiento porque representa un beneficio para el ser humano y por lo tanto nadie debería oponerse a aquello que es beneficioso para nosotros. Con bastante convencimiento Harris señala lo siguiente:

Existe una continuidad entre los daños y los beneficios, de manera que las razones que tenemos para evitar causar daños o crear a otros seres que nacerán con problemas serán una consecuencia de las razones que tenemos para beneficiar a otros si podemos. En resumen, decidir negar un beneficio es, en cierto modo, dañar al individuo que rechazamos mejorar. Tenemos razones para rechazar, crear $\mathrm{u}$ otorgar incluso beneficios triviales, así como razones para otorgar o no negar incluso pequeños beneficios. (Harris, 2017, p. 137)

Para Harris, existe un inmenso imperativo moral que justifica el mejoramiento con el fin de evitar daños y brindar beneficios. La legitimidad moral de este imperativo radica en aquellos mejoramientos que proporcionan beneficios o protegen de daños, y no tanto cuando sirven a los intereses de la igualdad de oportunidad o a la restauración del normal funcionamiento del organismo, que 
son las dos posturas que promueven Buchanan, Daniels, Brock y Wikler (2012) en la obra From Chance to Choice.

Frente a quienes consideran las tecnologías de mejoramiento como un deber moral, se sitúan quienes, como Jenny Krutzinna, han analizado el argumento transhumanista del deber moral situándolo en la estela del bienestarismo. Krutzinna parte de un principio humanista que se encuentra implícito en el welfarismo, según el cual la justificación de la bondad o la maldad de algo reside, en última instancia, en la contribución que representa para la vida humana y su calidad. Para el welfarismo no es necesario contar con una definición de vida buena en particular, sino que aquellos elementos que contribuyen beneficiosamente a la vida se identifican con facilidad, y por lo tanto se pueden encontrar bajo el paraguas del bienestar (Krutzinna, 2016, p. 529). Sin embargo, para Krutzinna la intuición no proporciona un fundamento lo suficientemente sólido para justificar el nivel de bienestar que algo proporciona. La persecución del bien se sitúa como el sentido teleológico del bienestarismo, aunque es necesario discutir las implicaciones que tiene este sentido.

Como se ha demostrado a lo largo de este trabajo, el desarrollo tecnológico y científico está protagonizando por un aumento considerable en las últimas décadas, lo que también va acompañado del crecimiento de una ambición positivista. En ese sentido, es fundamental plantear una reflexión ética que contribuya a la búsqueda de soluciones y al enriquecimiento de la discusión sobre las problemáticas. El motivo por el que es necesaria esta reflexión ética surge de la debilidad que presenta el enfoque welfarista para defender como un deber moral la mejora cognitiva mediante técnicas tecnocientíficas. Es decir, la normatividad que intenta proporcionar el welfarismo carece de solidez práctica. Por lo tanto, Krutzinna encuentra una necesidad imperante para discutir en qué consiste el bienestar. El concepto de bienestar basado en la teoría de los deseos presenta algunos problemas que surgen de la discusión entre lo objetivo y lo subjetivo (Krutzinna, 2016, p. 530).

\section{Objeto de discusión filosófica: bioconservadores y bioprogresistas}

No es demasiado el espacio bibliográfico que ocupa el fenómeno transhumanista en el ámbito filosófico español, pues como ha señalado Diéguez (2018) en un artículo publicado en el diario El País el 13 de septiembre de 2018, este es un objeto de estudio incipiente. Sin embargo, comienza a abrirse paso el estudio de este fenómeno, como atestigua la reciente publicación del libro de Diéguez: Transhumanismo. La búsqueda tecnológica del mejoramiento humano ilustra oportunamente este movimiento intelectual.

En lo que respecta al terreno filosófico, el transhumanismo ha estimulado la formulación de dos posiciones estrictamente diferenciadas y que representarán 
en las próximas décadas una importante pugna política en materia de regulación de las biotecnologías: bioconservadores y bioprogresistas. En el seno de la línea conservadora se sitúan aquellos que observan las tecnologías de mejoramiento desde la desconfianza, arrojando una mirada basada en el principio de precaución. Allen Edward Buchanan (2011) en su obra Beyond Humanity? The Ethics of Biomedical Enhancement, resume con claridad el eje discursivo sobre el que se elaboran los argumentos bioconservadores. Buchanam sostiene que el argumento conservador se sostiene sobre la existencia de una esencia humana que no puede ser alterada por una motivación caprichosa de perfeccionamiento. El organismo humano es producto de un ciclo evolutivo que se caracteriza por un complejo equilibrio que ha ido adquiriendo la actual forma a lo largo de la historia. En ese sentido, una alteración de la naturaleza humana representaría un desprecio al valor que representa la propia evolución obra del Maestro Ingeniero. Son varias las figuras que representan la postura conservadora en esta materia, como Jürgen Habermas (2009), Michael Sandel (2016), Francis Fukuyama (2002), George Annas (2017), Ryuichi Ida (2017), Steven J. Jensen y José Luis Widow (2018), aunque por motivo de espacio únicamente serán expuestos los postulados de Habermas, Sandel y Jensen y Widow.

Frente a los bioconservadores se sitúan aquellos que defienden la mejora tecnológica que impulsa al movimiento transhumanista, levantándose sobre las máximas de un perfeccionamiento ilimitado y un desafío a la muerte y el envejecimiento. Estas máximas serían realizables gracias a las impresionantes posibilidades que nos están brindando las biotecnologías. El control del envejecimiento y el rejuvenecimiento humano se convierte en un deber moral que debe ser asumido por la humanidad y reconocido en el contexto de los derechos humanos, como lo señalan José Luis Cordeiro y David Wood (2018, p. 17). Entre los bioprogresistas podemos encontrar a Peter Sloterdijk, Ray Kurzweil o Nick Bostrom.

Los avances que el campo de la biotecnología ha experimentado en las últimas décadas han provocado el surgimiento de debates en el seno de numerosas esferas como la jurídica, la psicológica, la científica, la filosófica, etc. Son variadas las posturas respecto a la biotecnología; por un lado, se encuentran aquellos puntos de vista que observan estos mecanismos tecnocientíficos como una forma sofisticada de eugenesia que tendrá un gran impacto; por otro lado, se sitúan los que defienden con convicción que representa la solución a serios problemas que enfrenta la humanidad y que son de diversa índole.

\section{Jürgen Habermas y la crítica a la manipulación "caprichosa"}

Habermas ha participado en este debate con la publicación de El futuro de la naturaleza humana. ¿Hacia una eugenesia liberal?, obra que también representa una tesis opuesta a la expuesta por Peter Sloterdijk (2001) en Normas para el parque humano. A Habermas le interesa principalmente la investigación con 
células madre y el diagnóstico genético preimplantacional (DGP). Este filósofo trata de reflexionar acerca de las consecuencias que tendrá para la modernidad política las técnicas de manipulación de la especie que representan las diversas formas de intervención genética. El texto sobre el futuro de la naturaleza humana en el que Habermas esboza su reflexión acerca de la biotecnología es de carácter político, como señala Eduardo Mendieta (2002, p. 94), ya que las referencias filosóficas que en él se hace son exclusivamente para reforzar argumentaciones políticas. Para el filósofo alemán no ha existido un profundo debate público sobre las implicaciones de los DGP, ya que la ciudadanía se ha dejado llevar de forma acrítica por la oferta mercantil y la libertad individual promovida por el credo liberal. Frente a esta posición acrítica es fundamental pensar acerca de la necesidad de normativizar en este terreno, ya que, si no se plantea una seria reflexión pública, las biotecnologías tendrán efectos dañinos sobre nuestra consideración de la condición humana y nuestra cultura política. En ese sentido, Habermas muestra su rotunda oposición a la eugenesia liberal.

La preocupación habermasiana es de carácter moral, ya que el centro de la cavilación se enfoca en la autocomprensión moral. Habermas no se opone con rotundidad a todas las manipulaciones genéticas, ya que solo le parecen aceptables aquellas que persiguen como finalidad la erradicación de enfermedades. Para Habermas, la manipulación genética representa un uso instrumental de los embriones y por lo tanto una acción contraria el postulado kantiano que promovía el tratamiento del ser humano como un fin en sí mismo y no como un medio. En ese sentido, la línea argumentativa del filósofo alemán se encuentra muy cercana a la de la Iglesia católica, al considerar al embrión como una persona humana en potencia desde el momento de la concepción.

\section{Michael Sandel y la ética de la gratitud con lo dado}

En su obra Contra la perfección. La ética en la era de la ingeniería genética, Sandel presenta su postura acerca de las implicaciones éticas de las biotecnologías, lo que ha despertado un gran interés en el ámbito académico internacional y en las instituciones políticas de los EE. UU., donde Sandel participó junto a Fukuyama en el comité de ética creado en el 2002 bajo la presidencia de George W. Bush. Lo que despierta el interés en Sandel es la falta de comprensión moral que presentan los seres humanos frente a los profundos avances de las biotecnologías, es decir, que la ciencia avanza a una mayor rapidez que nuestra comprensión moral (Sandel, 2016, p. 75). El argumento bioconservador que se sostiene sobre la autonomía y la libertad de los hijos con respecto a los padres que han decidido manipular sus genes no es del todo convincente para Sandel, pues da por sentado que si los padres no deciden sobre el diseño del hijo, entonces el hijo será inevitablemente libre, algo que no es cierto, ya que nadie elige su herencia genética, que depende de la llamada "lotería genética" (Sandel, 2016, p. 76).

La idea central del argumento de Sandel para el cuestionamiento del transhumanismo es el paso de una ética del agradecimiento de lo que nos es dado, 
parecida a la que sostiene Ryuichi Ida (2017), a una ética del dominio sobre la naturaleza por parte de un hombre prometeico. Como señala Ferry, cuando se habla de aquello que nos es dado no necesariamente se remite a una autoridad religiosa, sino a una cuestión secular, a un "principio de donación exterior y superior al hombre" (Ferry, 2017, p. 90), que puede ser la misma naturaleza. Esta relación azarosa y misteriosa es la que se deja a un lado en el transhumanismo bajo las pretensiones de dominio sobre la naturaleza. Para Sandel, este carácter prometeico y dominador que caracteriza al transhumanismo desembocaría en la transformación de tres aspectos claves de nuestra condición moral: la humildad, la responsabilidad y la solidaridad (Sandel, 2016, pp. 91-92).

\section{Steven J. Jensen y José Luis Widow y la crítica naturalista}

En el artículo Unnatural Enhancements Steven J. Jensen y José Luis Widow critican aquellas técnicas de mejoramiento que representan una amenaza para el orden natural de la naturaleza. Los autores diferencian entre mejoras terapéuticas y no terapéuticas, entendiendo que hay mejoras que son pertinentes y otras no, en función del respeto al orden de la naturaleza.

Partiendo de la clasificación que establece Lisbeth Nielsen en función de la noción de naturaleza, llevan a cabo su posicionamiento, dicha clasificación se establece de la siguiente manera: 1) un estado general de las cosas que podría denominarse "madre naturaleza"; 2) la esencia de una cosa, en particular la esencia de los seres humanos; y 3) lo que está libre de la intervención humana, es decir, lo natural en oposición a lo artificial (Witthøfft, 2011, p. 350). La preocupación de Jensen y Widow se centra en la relación que como humanos establecemos con la naturaleza. Tomando como referencia en su fundamentación la teoría aristotélica de la naturaleza, afirman que esta se caracteriza un sentido teleológico y dinámico, distanciándose de ese modo de las concepciones que consideran a la naturaleza como una entidad estática.

La naturaleza tiene un sentido teleológico, está orientada a un fin concreto, si esta tendencia a una finalidad concreta sufre una alteración, estaríamos frente a una acción antinatural que dificulta el despliegue de la naturaleza. La desviación de aquello que no es dado por la naturaleza es considerada como una consecuencia fruto de una acción antinatural. En ese sentido, el transhumanismo no estaría promoviendo el orden natural, sino más bien respondiendo a una satisfacción de carácter privado del ser humano (Witthøfft, 2011, p. 352).

Por lo tanto, una modificación de las tendencias propias del orden de la naturaleza representaría una alteración de curso teleológico. Esta alteración del orden es antinatural, y cualquier mecanismo que represente una alteración para dicho orden, serían elementos antinaturales. En ese sentido, todos aquellos mecanismos tecnocientíficos que promuevan una alteración del orden natural o una sustitución de este, serían considerados por Jensen y Widow como antinaturales. 
Por lo tanto, las tesis transhumanistas serían antinaturales porque responden a un deseo privado innecesario, y supone una alteración para la naturaleza.

\section{Peter Sloterdijk y la antropotécnica}

Peter Sloterdijk es de esos filósofos atrevidos en sus planteamientos y así lo demuestra en Normas para el parque humano. Esta obra es fruto de un texto que expuso en un seminario a los pocos años de la muerte de Emmanuel Lévinas, y que despertaría un interesante debate filosófico en los últimos años, sobre todo con Jürgen Habermas. La conferencia tuvo lugar el 17 de julio de 1999 en el castillo de Elmau, en Baviera, con motivo del simposio internacional "Jenseits des Seins /Exodus from Being/ Philosophie nach Heidegger". Sloterdijk formula esta reflexión como una respuesta a la Carta sobre el humanismo de Heidegger. Lleva a cabo un diagnóstico sobre la capacidad crítica del humanismo que entiende como tradicional, que ha desembocado, según él, en un naufragio como escuela y proyecto de domesticación del género humano. Sloterdijk nos invita a pensar la posibilidad de emprender nuevos caminos vinculados a un aprovechamiento de la tecnología para la elaboración de un nuevo relato humano.

A partir de las lecturas del Político de Platón y de la Carta sobre el humanismo de Heidegger, Sloterdijk considera que se debe superar el proyecto humanista de tradición literaria y plantear un nuevo relato que esté contextualizado en la era tecnológica, concretamente en el tiempo de la ingeniería genética. En su reflexión pone el acento en el concepto de domesticación, entendido como la técnica por la cual el ser humano ha conseguido, a través de la educación y la cultura, establecer una clara diferenciación entre los del mismo género, algo que él denomina como antropotécnica. El universo de posibilidades que nos brinda la tecnología, y concretamente la biotecnología, representa una vía pragmática desde la que es posible desarrollar nuevas antropotécnicas. Este universo de posibilidades que nos es brindado demanda de un código de las antropotécnicas donde el ser humano participe consciente y activamente.

\section{Raymond Kurzweil y la integración con la tecnología}

Raymond Kurzweil es otro de los íconos de ese transhumanismo de corte tecnocientífico inspirado en el campo de la IA y la ingeniería computación en general. En su obra La singularidad está cerca promueve el concepto de singularidad que ha servido para impulsar un proyecto universitario muy prometedor como la Singularity University, y que en su página web se presenta de la siguiente manera:

Preparación de líderes mundiales y organizaciones para el futuro: explore las oportunidades e implicaciones de las tecnologías exponenciales y conéctese a un ecosistema global que está configurando el futuro y resolviendo los problemas más urgentes del mundo. (Singularity University, 2019, párr. 1-2) 
Kurzweil utiliza el concepto de singularidad para referirse al momento en el acontezca una explosión de superinteligencia capaz de perfeccionarse a sí misma y de fabricar otros sistemas inteligentes al margen de la intervención humana, y así sucesivamente por medio de un crecimiento exponencial que derivará en una entidad global con una inteligencia artificial y superior a la de los seres humanos. El término "singularidad" se emplea en el ámbito de la Teoría General de la Relatividad para designar un sistema o estado en el que no se da lugar a la aplicación de leyes físicas conocidas. Por lo tanto, este no es un término propio del campo transhumanista, sino que más bien se emplea como una mera analogía para referirse a lo discontinuo y lo impredecible, algo que tiene que ver con la entrada en un escenario desconocido y poshumano.

Hay un hecho muy importante para entender mejor el pensamiento transhumanista de Kurzweil. Es un autor agnóstico en lo que respecta a las creencias metafísicas, y lo es porque considera que la argumentación teísta sobre un más allá representado por un ser divino no es descartable, y de la misma manera tampoco sería descartable el ateísmo. Su posición se debe a la formación religiosa que recibió durante su juventud, centrada principalmente en el conocimiento de varias religiones. En ese sentido, ese hecho formativo en su etapa de juventud lo marcará para toda la vida y sentará las bases en su pensamiento para cultivar la idea de convergencia. Kurzweil ha sabido aprovechar muy bien la idea de convergencia para entender que existe la posibilidad de imaginar más allá de la naturaleza humana entendida biológicamente y trascender hacia un espacio tecnológico, desafiando así las fronteras entre lo biológico y lo tecnológico.

Anticipándose a posibles críticas, Kurzweil sostiene que la trascendencia desde lo biológico a lo tecnológico no implica una desaparición de la humanidad a expensas de las máquinas con superinteligencia. Por ello, su propuesta se centra en nuestra integración con la máquina, en un proceso de síntesis consistente en la transferencia de nuestra mente en una de ellas. Dicho esto, la singularidad podría ser reconocida desde dos perspectivas, por un lado, como la creación de sistemas con superinteligencia; por otro lado, como el fortalecimiento de la inteligencia humana hacia niveles poshumanos gracias a la integración con la tecnología. En ese sentido, la singularidad se presenta como una nueva etapa en el proceso evolutivo que se verá motivada por la necesidad de trascender:

La singularidad denota un evento que tendrá lugar en el mundo material, el inevitable siguiente paso en el proceso evolutivo que empezó con la evolución biológica y que se ha extendido mediante la evolución tecnológica dirigida por los humanos. Sin embargo, es precisamente en el mundo de la materia y de la energía donde nos enfrentamos a la trascendencia, una de las connotaciones principales de lo que la gente llama espiritualidad. Consideremos la naturaleza de la espiritualidad en el mundo físico. (Kurzweil, 2017, pp. 443-444)

A pesar de las críticas que los defensores de la singularidad han recibido, se siguen situando en la senda ideológica de un cambio tecnológico radical que 
significará un verdadero cambio de paradigma para la vida humana en varias de sus dimensiones. Las tesis de Kurzweil han levantado ampollas en los laboratorios de IA, aunque también cuenta con importantes seguidores.

\section{Nick Bostrom y la apertura de posibilidades}

En su texto In Defense of Posthuman Dignity, Bostrom formula una crítica sobre los posicionamientos bioconservadores de figuras conocidas como las de Leon Kass, Francis Fukuyama, George Annas, Wesley Smith, Jeremy Rifkin y Bill McKibben. El fundador del Future of Humanity Institute afirma que la naturaleza nos brinda en ocasiones regalos envenenados como el cáncer, y que estamos obligados moralmente a hacer un uso de la tecnología para el beneficio de nuestra especie. Además, una de las típicas críticas que los bioconservadores dirigen contra el transhumanismo se centra en la posibilidad de que se genere un escenario en el que los poshumanos atenten contra la vida de los humanos. En ese sentido, Bostrom reconoce el importante poder que se encuentra implícito en las biotecnologías, aunque afirma que la defensa de las biotecnologías para mejorar las condiciones de vida de los seres humanos no es incompatible con estar a favor de una política reguladora de estas actividades (Bostrom, 2007, p. 206).

El postulado transhumanista de Bostrom no se caracteriza por el optimismo tecnológico, ya que reconoce que el enorme potencial tecnológico que nos depara el futuro también puede ser empleado de mala manera, y por lo tanto provocar un daño enorme, que va desde la extinción de la vida inteligente hasta el incremento de las desigualdades sociales. Los riesgos que implica la tecnología en el ámbito del mejoramiento humano deben ser tenidos muy en cuenta para que los proyectos que se impulsen lo hagan con precaución y teniendo en cuenta diversidad de factores de impacto (Bostrom, 2005, p. 4). Para promover los valores transhumanistas y las actividades de mejoramiento de la especie humana, es fundamental asumir tres condiciones básicas: la seguridad global, la necesidad de un desarrollo tecnológico que vaya acompañado de crecimiento económico y una apertura en el acceso a los beneficios tecnológicos para que no sean exclusivos de las clases adineradas (Bostrom, 2005, pp. 9-11).

El ser humano es un ser que se caracteriza por su limitación natural, pues existen una serie de pensamientos, sentimientos, experiencias, etc., a las que tendríamos la posibilidad de acceder si superáramos las limitaciones que nos impone nuestra naturaleza biológica. Es asombroso pensar que existe un universo de posibilidades más allá de lo que conocemos. Ese universo de posibilidades es posible disfrutarlo por medio de una nueva realidad poshumana, que trascienda lo transhumano. Aquí, Bostrom establece una clara diferenciación entre lo transhumano y lo poshumano, considerando al primero como un momento de transición caracterizado por mejoras moderadas; en cambio, el poshumano va más allá del estado de transición y se sitúa en una nueva esfera. En lo que concierte a la accesibilidad de la realidad posible, Bostrom utiliza la siguiente 
figura para ilustrar gráficamente las parcelas de dominio de los animales, los humanos, los transhumanos y los poshumanos (Bostrom, 2005, p. 5).

Figura 1. Transhumanist values. Philosophy Documentation Center

The Space of Possible Modes of Being

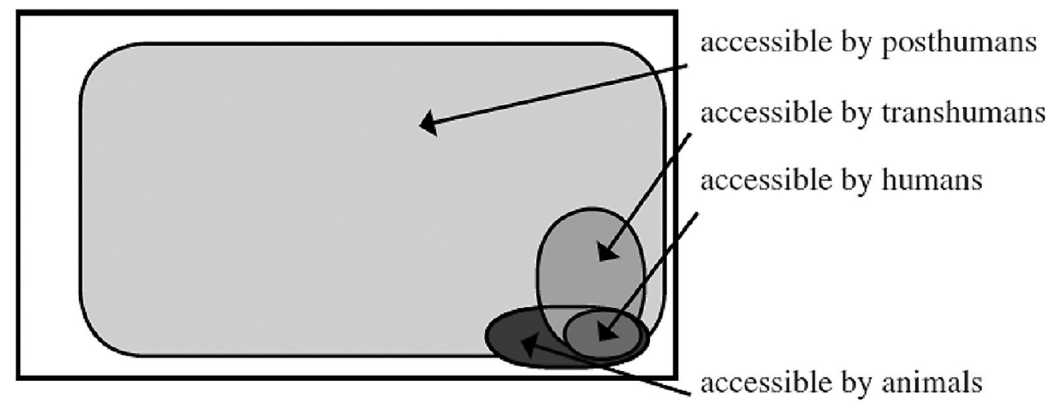

Fuente: Bostrom, 2005

Como se ha señalado en el apartado 3.4, Bostrom orienta las prioridades transhumanistas a partir de tres ejes (Bostrom, 2008, p. 107):

- Salud: capacidad de mantenerse completamente sano, activo y productivo, tanto mental como físicamente.

- Cognición: capacidades intelectuales generales, como la memoria, el razonamiento deductivo y analógico, y la atención, así como facultades especiales tales como la capacidad de comprender y apreciar la música, el humor, el erotismo, la narración, la espiritualidad, las matemáticas, etc.

- Emotividad: capacidad de disfrutar la vida y responder con un efecto apropiado a las situaciones de la vida y a otras personas.

\section{La problemática de los factores de pertenencia social y de identidad}

El fenómeno transhumanista tiene importantes implicaciones políticas, pues es una actividad humana que necesariamente se desarrolla dentro de una comunidad concreta. Según Christine Overall (2009), la opinión acerca de que la ética dedicada a la reflexión en el campo del mejoramiento no debería centrarse únicamente en temas relacionados con los riegos, los beneficios, los costes o la autenticidad de la especie, sino que también debería incorporar a la discusión aspectos de índole social y política (Overall, 2009, p. 327). Las tecnologías de mejoramiento se encuentran estrechamente vinculadas con cuestiones políticas de poder, por ejemplo, en temas de propiedad de los medios tecnológicos, 
oportunidades de acceso a los mecanismos de mejora que se encuentran dentro del ámbito de pertenencia a una clase social, y nuevas formas de opresión que se discutirán más adelante.

Cuando los mecanismos tecnológicos de mejoramiento son desplegados, pueden generar problemas de opresión entre grupos con diferente identidad y también provocar claras relaciones de desigualdad. El uso de la tecnología para mejorar la vida de los individuos tendría que ser algo positivo para una sociedad, sin embargo, esas mejoras podrían ser exclusivas de determinados grupos y generar dinámicas de poder que dificultaran las oportunidades de acceso a otros grupos, fomentando de ese modo estrategias de exclusividad. Estas estrategias de exclusividad obstaculizarían el establecimiento de relaciones equitativas e inclusivas entre los diversos grupos que conforman una sociedad, por lo tanto, nos estaríamos enfrentando a un problema de carácter político.

Los grupos de pertenencia social que configuran nuestra identidad pueden ser de diversa índole: sexo, género, etnia, clase económica, raza, etc. En ese sentido, la identidad influye sobre nuestra posición dentro de una sociedad y por lo tanto en el aprovechamiento de oportunidades que las instituciones nos brindan. El acceso a las tecnologías está fuertemente marcado por la pertenencia a un grupo social, por lo que el mejoramiento humano también pueda ser un factor de desigualdad de oportunidades. Un gobierno puede legislar en materia de mejoramiento, liberalizar ese sector al extremo, facilitando mecanismos políticos y jurídicos para hacer uso de la libertad, si bien eso no garantizaría bajo ningún concepto que todos los individuos de una sociedad tuvieran igual acceso a las oportunidades de estas tecnologías. Esto se debe esencialmente a lo que se ha mencionado con anterioridad, a saber, el grupo social de pertenencia. El derecho del libre uso y acceso a los sistemas de mejoramiento no garantiza que todos los individuos de una sociedad puedan ejercer ese derecho, ya que estamos hablando de cuestiones que requieren de cierta posición socioeconómica. Por lo tanto, existe un importante condicionamiento a partir de la pertenencia a un grupo social, aunque este fenómeno de condicionamiento social no es nada nuevo. La pertinencia de analizar el impacto del condicionamiento social surge a raíz del nuevo escenario tecnológico al que nos enfrentamos. Bostrom es consciente de la problemática que suscita el mejoramiento humano en términos de desigualdad y sostiene lo siguiente:

Los genéticamente privilegiados puede convertirse en genios eternos, sanos, súper genios de una belleza física impecable, dotados de un ingenio deslumbrante y un sentido del humor despectivamente autodestructivo, irradiando calidez, encanto empático y confianza relajada. Los no privilegiados se mantendrían como las personas hoy en día, pero tal vez privados de su respeto por sí mismo y sufriendo episodios ocasionales de envidia. La movilidad entre las clases baja y alta podría desparecer y un niño nacido de padres pobres, sin mejores genéticas, podía encontrar que es imposible competir con éxito contra los súper hijos de los ricos. Incluso si no hubo discriminación o explotación de la clase baja, todavía hay 
algo inquietante sobre la perspectiva de una sociedad con tales desigualdades extremas. (Bostrom, 2004, p. 502)

Razonamientos como el Bostrom u Overall son los que pueden llevarnos a plantear la necesidad de discutir el fenómeno del transhumanismo en términos políticos incorporando criterios de responsabilidad y justicia. Nos enfrentamos a una nueva forma de desigualdad fruto de la tecnología. La tecnología en sí misma no tiene un carácter negativo, sino que depende de los humanos el hacer un buen uso de ella para gestionar la vida de las sociedades aspirando a mayores cuotas de felicidad. En ese sentido, la estrategia a adoptar frente a la desigualdad no debería ser prohibir los mecanismos de mejoramiento, sino diseñarlos de tal manera que adopten formas inclusivas.

\section{Ua necesidad de una profunda reflexión ética}

La entrada de la tecnología a gran escala en el ámbito social nos invita a discutir las implicaciones que existen para nuestras vidas. Los impactos de las tecnologías son variados, pues dependen de la finalidad y el contexto en el que se encuentren insertadas y tienen un gran potencial transformador, como señala Kevin Kelly:

El cambio es inevitable. Ahora nos parece que todo es transformable y está sujeto a transformaciones, aunque gran parte de estos cambios sean imperceptibles. [...] En el núcleo de todo cambio significativo de nuestras vidas hay algún tipo de tecnología. La tecnología conlleva la aceleración de la humanidad. Gracias a la tecnología, todo lo que hacemos se encuentra siempre en proceso de transformación. Todas las clases de cosas se convierten en algo nuevo, mientras pasan de "podrían" a "son". Todo se encuentra en flujo permanente. Nada está totalmente terminado. Nada está hecho. Este cambio constante es el eje en torno al cual gira el mundo moderno. (Kelly, 2017, p. 5)

Jean Marc Lévy-Leblond destaca que la ciencia es una actividad que cae bajo el paraguas de las actividades morales, porque se encuentra insertada en contextos sociales que son susceptibles de reflexión en torno al principio de responsabilidad. El conocimiento científico es susceptible de una consideración ética donde entra en juego la reflexión sobre las implicaciones que tiene para la vida de los seres humanos y la biosfera. A colación de la supuesta neutralidad axiológica del conocimiento científico, es importante mencionar tres puntos que LévyLeblond (1975) destaca para cuestionar la idea de neutralidad de la ciencia que el positivismo ha promovido en su discurso:

- Los científicos que suelen rechazar la responsabilidad de aquellas consecuencias nefastas por su trabajo, reclaman, por otro lado, una serie de elogios y reconocimientos por los efectos positivos, con el fin de ocultar las implicaciones negativas. 
- La ciencia no puede ser un conocimiento neutro porque no se sitúa al margen de influencias externas, ya que existen infinidad de intereses que ejercen su influencia sobre las investigaciones.

- Los científicos no se encuentran al margen de la sociedad, y por lo tanto la ciencia no puede escapar de la influencia directa de las condiciones del contexto económico, político y social.

Por algunos motivos que pueden ser económicos o estrictamente tecnocientíficos, en ocasiones se torna complejo llevar a cabo un ejercicio de observación sobre las implicaciones que ciertos mecanismos presentan. La lógica económica normalmente activa la maquinaria de la falta de cuestionamiento bajo la afirmación de que la rentabilidad económica es un motivo más que suficiente como para no ser cuestionado ni relegado a un segundo plano.

Además, la formación ética de los especialistas del campo tecnológico suele brillar por su ausencia. Ha sido en los últimos años cuando se ha introducido la asignatura de ética en algunos centros académicos, con el objetivo de brindar herramientas de orientación a los futuros profesionales del sector para que configuren de un mejor modo su actividad. En ese sentido, tanto la lógica económica como la falta de formación ética de los profesionales de la tecnología dificultan la identificación de las implicaciones éticas en este campo y también representan un claro obstáculo para incorporar criterios de responsabilidad a la acción tecnológica.

En el ámbito de las tecnologías de mejoramiento es fundamental introducir principios democráticos para dotar a este campo de un espíritu comprometido con el bienestar general de la sociedad, existiendo de ese modo un claro compromiso con los Objetivos de Desarrollo Sostenible (ODS) y los Derechos Humanos (DD. HH.). A colación de la contextualización de la democracia dentro de un escenario tecnológico de aumento de posibilidades, Miguel Ángel Quintanilla diferencia entre una democracia tecnológica mínima y una democracia tecnológica plena, dentro de las cuales identifica varios puntos cardinales (Quintanilla, 2002, pp. 640-647). En el interior de la democracia tecnológica mínima pueden ser identificados los siguientes puntos:

- Derecho de todos los ciudadanos a participar en las decisiones sobre el uso de las posibilidades tecnológicas en asuntos de interés público.

- Derecho de todos los ciudadanos de acceder al conocimiento técnico y de contar con el juicio de los expertos como elemento fundamental para conformar la opinión pública y participar en las decisiones políticas sobre asuntos técnicamente complejos.

- No convertir en problemas políticos aquellos asuntos para los que existen soluciones técnicas solventes y contrastadas. 
En cuanto a la democracia tecnológica plena destaca principalmente el siguiente aspecto:

- El derecho de todos los ciudadanos a acceder a todo el conocimiento tecnológico relevante para la toma de decisiones en asuntos de interés público y a participar en el diseño, evaluación y control del desarrollo tecnológico.

La propuesta de Antonio Diéguez (2017) para enfrentar los retos que nos plantea el transhumanismo es ante todo reflexiva, ya que sugiere un alejamiento de aquellas posturas que se caracterizan por la tecnofobia y por la tecnofilia ciega y carente de crítica. En ese sentido, y siguiendo a Diéguez, no sería sensato ni aceptar ni rechazar enteramente los proyectos transhumanistas, sino que más bien deberían ser sometidos a una evaluación exhaustiva teniendo en cuenta los aspectos particulares, es decir, la casuística. A propósito de esto, Diéguez sostiene lo siguiente, "es primordial, por tanto, evitar el error común de realizar juicios generales y definitivos, de lanzar condenas o alabanzas globales" (Diéguez, 2017, p. 194). Es muy posible que algunas tecnologías de mejoramiento favorezcan una mejora de la vida humana, aunque otras podrían resultar horrorosas.

Alfredo Marcos (2018) critica la interpretación que Diéguez realiza del pensamiento de Ortega en el contexto del transhumanismo. Reconoce la sensatez del planteamiento de Diéguez, aunque afirma que la concepción platónica de la naturaleza humana de la que parte y las ideas de Ortega resultan inoperantes porque están fuertemente marcadas por una antropología antinaturalista y, en cierta medida, limitadas. La contribución que Ortega hace a la filosofía de la técnica es muy interesante, se caracteriza por una antropología con un marcado carácter existencialista, si bien en ocasiones destaca por el antinaturalismo. El recurso orteguiano es interesante, pero ante el fenómeno tecnológico, y más concretamente ante las tesis transhumanistas, no es más que una fuente secundaria (Marcos, 2018, p. 11).

La visión naturalista del ser humano que Ortega reconoce, y que posteriormente parece ser que asumirá Diéguez, es limitada, ya que identifica al ser humano exclusivamente dentro del ámbito de la naturaleza. Frente a esa visión de la naturaleza humana empobrecidamente biologicista que Ortega rechaza, se encuentra una visión mucho más integral de inspiración aristotélica que Marcos analiza. Marcos cree que existen buenas razones para aceptar la existencia de una naturaleza con implicaciones normativas que van más allá del concepto de naturaleza humana que los transhumanistas, Ortega y Diéguez rechazan. En cambio, es precisamente este concepto de naturaleza humana más integral el que podría permitirnos reflexionar sobre la valoración de las particularidades que tanto reivindica Diéguez, ya que nos estaría dotando de criterios para poder discernir con recursos mucho más amplios. 


\section{Conclusión}

Como se ha podido comprobar a lo largo de este trabajo, el proyecto transhumanista es sumamente ambicioso y plantea diversas estrategias desde las que desafiar la condición humana. Ha provocado un profundo debate en el seno de la filosofía, donde bioconservadores y bioprogresistas discuten sobre las problemáticas que giran en torno a las tecnologías de mejoramiento y su impacto en la sociedad.

Es necesario generar un debate público en torno al proyecto transhumanista, para de ese modo abordar las controversias que subyacen en su puesta en marcha. Los diversos actores que conforman la compleja realidad que marca el rumbo de la sociedad, Estado, centros de conocimiento, sector privado y organizaciones de la sociedad, se ven empujadas a participar en un debate enriquecedor en el que se traten las problemáticas que generan las tecnologías de mejoramiento. El cultivo de una cultura de diálogo multidisciplinar es fundamental para valorar el impacto de estas tecnologías y también para generar conocimientos que sean innovadores. Además, es fundamental que también sean tomados en cuenta los Derechos Humanos y los Objetivos de Desarrollo Sostenible, con el fin de que las tecnologías contribuyan a hacer un mundo mejor. Precisamente en ese punto reside el desafío que nos depara el transhumanismo, en saber un uso responsable de la tecnología para hacer un mundo mejor para todos.

\section{Referencias}

Annas, G. J. (2017). Health and Human Rights: Of Bridges and Matrixes. Am J Bioeth, 17(9), 13-15.

Bostrom, N. (2003a). The Transhumanist FAQ: A General Introduction. World Transhumanist Association.

Bostrom, N. (2003b). Transhumanist values. In F. Adams (ed.), Ethical Issues for the 21st Century (pp. 3-14). Philosophical Documentation Center Press.

Bostrom, N. (2004). Human Genetic Enhancements: A Transhumanist Perspective. The Journal of Value Inquiry, 37, 493-506. DOI: https://doi. org/10.1023/b:inqu.0000019037.67783.d5

Bostrom, N. (2005). Transhumanist values. Philosophy Documentation Center. Recuperado de https://nickbostrom.com/ethics/values.html

Bostrom, N. (2007). In Defense of Posthuman Dignity. Bioethics, 19(3), 202-214. 
Bostrom, N. (2008). Why I Want to be a Posthuman When I Grow Up. In B. Gordijn \& R. Chadwick (eds.), Medical Enhancement and Posthumanity (pp. 107-137). London: Springer. DOI: https://doi.org/10.1007/978-1-4020-8852-0 8

Buchanan, A. E. (2011). Beyond Humanity?: The Ethics of Biomedical Enhancement. Oxford: Oxford University Press.

Buchanan, A., Brock, D. W., Daniels, N. y Wikler, D. (2012). From Chance to Choice. Genetics $\mathcal{E}$ Justice. Cambridge: Cambridge University Press.

Cordeiro, J. L. y Wood, D. (2018). La muerte de la muerte. La posibilidad científica de la inmortalidad física y su defensa moral. Barcelona: Ediciones Deusto.

De Grey, A. (2013). El fin del envejecimiento. Berlín: Lola Books.

Diéguez, A. (2017). Transhumanismo. La búsqueda tecnológica del mejoramiento humano. Barcelona: Herder. DOI: https://doi.org/10.21555/top.v0i56.1025

Diéguez, A. (2018). Transhumanismo y filosofía. El País. Recuperado de https:// elpais.com/elpais/2018/09/12/opinion/1536752872 112358.html

Feito, L. (2013). El debate ético sobre la mejora humana. Diálogo filosófico, 8, 45-51.

Ferry, L. (2017). La revolución transhumanista: cómo la tecnomedicina y la uberización del mundo van a transformar nuestras vidas. Madrid: Alianza Editorial.

Fukuyama, F. (2002). El fin del hombre: consecuencias de la revolución biotecnológica. Barcelona: Ediciones B.

González F. J. (2010). Transhumanismo (humanity +). La ideología que nos viene. Recuperado de https://www.academia.edu/3621186/Transhumanismo humanity_La_ideolog\%C3\%ADa_que nos viene

Habermas, J. (2017). El futuro de la naturaleza humana: ¿Hacia una eugenesia liberal? Barcelona: Paidós.

Harris, J. (2017). Los mejoramientos son una obligación moral. En N. Bostrom y J. Savulescu (eds.), Mejoramiento humano (pp. 137-161). España: Teell Editorial.

Humanity + (2018). Transhumanist Declaration. Recuperado de https://humanityplus.org/philosophy/transhumanist-declaration/

Ida, R. (2017). ¿Deberíamos mejorar la naturaleza humana? Un interrogante planteado desde una perspectiva asiática. En N. Bostrom y J. Savulescu (eds.), Mejoramiento humano (pp. 63-74). España: Teell Editorial. 
Jensen, S. J. y Widow, J. L. (2018). Unnatural Enhancements. SAGE journals, 83(4), 347-363.

Kelly, K. (2017). Lo inevitable. Entender las 12 fuerzas tecnológicas que configurarán nuestro futuro. España: Editorial TEELL.

Krutzinna, J. (2016). Can a welfarist approach be usted to justify a moral duty to cognitively enhance children? Bioethic, 30(7), 528-535. DOI: https://doi. org/10.1111/bioe.12244

Kurzweil, R. (2017). La singularidad está cerca. Berlín: Lola Books.

Lévy-Leblond, J. M. (1975). La ideología delen la física contemporánea y ostros ensayos. Barcelona: Editorial Anagrama.

Marcos, A. (2018). Bases filosóficas para una crítica al transhumanismo. Revista Artefactos, 7(2), 107-125. DOI: https://doi.org/10.14201/art201872107125

Mendieta, E. (2002). El debate sobre el futuro de la especie humana: Habermas critica la eugenesia liberal. Isegoría, 27, 91-114. DOI: https://doi.org/10.3989/ isegoria.2002.i27.556

More, M. (1990). Transhumanism: Toward a Futurist Philosophy. Extropy, 6, 6-12. Recuperado de http://fennetic.net/irc/extropy/ext6.pdf

Ortega y Gasset, J. (2004). Meditación de la técnica y otros ensayos sobre ciencia y filosofía. Madrid: Revista de Occidente.

Overall, C. (2017). Tecnologías de mejoramiento de la vida: el significado de la pertenencia a una categoría social. En N. Bostrom y J. Savulescu (eds.), Mejoramiento humano (pp. 339-353). España: Teell Editorial.

Quintanilla, M. A. (2002). La democracia tecnológica. Arbor, 637-652.

Sandel, M. (2016). Contra la perfección: la ética en la era de la ingeniería genética. Barcelona: Marbot Ediciones.

Savulescu, J. (2012). ¿Decisiones peligrosas? Una bioética desafiante. Madrid: Tecnos.

Singularity University (2017). Portada de la web principal. Recuperado de: https:// su.org/

Sloterdijk, P. (2001). Normas para el parque humano: una respuesta a la carta sobre el humanismo de Heidegger. Madrid: Siruela. 
Tännsjö, T. (2017). El mejoramiento médico y los valores del deporte de élite. En N. Bostrom y J. Savulescu (eds.), Mejoramiento humano (pp. 327-338). España: Teell Editorial.

Witthøfft, L. (2011). The Concept of Nature and the Enhancement Technologies Debate. En J. Savulescu et al., (eds.), Enhancing Human Capacities. Chichester: Wiley-Blackwell. 